\title{
The Role of Verbal Working Memory in Children's Sentence Comprehension A Critical Review
}

\section{Evan Kidd}

This article reviews research that has investigated the role of verbal working memory (VWM) in sentence comprehension in both typical and atypical developmental populations. Two theoretical approaches that specify different roles for VWM in sentence comprehension are considered: (i) capacity-limit approaches, which treat VWM as a theoretical primitive that causally constrains language processing and acquisition, and (ii) the experience-based approach, which argues that VWM is an emergent property of long-term linguistic knowledge. The empirical literature relevant to these different approaches is then reviewed. Although there has been considerable recent research on the topic, it is concluded that the current role of working memory in sentence comprehension in development is unclear, calling for a greater number of controlled systematic developmental studies on the topic. Keywords: language acquisition, sentence comprebension, verbal working memory

$\mathbf{W}$ ORKING MEMORY (WM) has long been argued to play a significant role in language acquisition. This review is concerned with verbal working memory (VWM) and the role it plays in children's sentence comprehension. "Verbal working memory" is defined as the component of memory that stores and manipulates verbal information (e.g., the central executive component of the [Baddeley, 2007] WM model). It can be dis-

\section{Autbor Affiliation: Australian National University, Canberra, Australia.}

Many thanks to Lisa Archibald, Nicki Nelson, and two anonymous reviewers for belpful comments on a previous draft.

The author has disclosed that he has no significant relationships with, or financial interest in, any commercial companies pertaining to this article.

Corresponding Author: Evan Kidd, PhD, Research School of Psychology (Building 39), Australian National University, Canberra 0200, Australia (evan .kidd@anu.edu.au).

DOI: $10.1097 /$ TLD.0b013e31829d623e tinguished from phonological or verbal shortterm memory (STM), which is typically measured using nonword repetition tasks or list recall, for which there is a long history of research in the language acquisition literature (e.g., Gathercole, Willis, Emslie, \& Baddeley, 1992; Melby-Lervåg et al., 2012; Stokes \& Klee, 2009; Willis \& Gathercole, 2001). In contrast, comparably less research has been conducted on the role of VWM in language acquisition, despite the fact that this topic has received considerable attention in adult psycholinguistic research (e.g., Just \& Carpenter, 1992; Lewis, Vasishth, \& Van Dyke, 2006; MacDonald \& Christiansen, 2002; Waters \& Caplan, 1996). The research that exists has produced fairly inconsistent results and has been hampered by the largely implicit assumption that VWM causally constrains but is conceptually independent from long-term linguistic knowledge.

This article aims to (i) outline explicitly the main theoretical divisions in the study of VWM, (ii) survey the current research on the role of VWM in sentence comprehension in both typical and atypical developmental 
populations, and (iii) identify future directions for research.

\section{BACKGROUND}

Working memory is a complex construct describing the concurrent maintenance and manipulation (i.e., processing) of information (Oberauer \& Lewandowsky, 2010). Several theoretical models exist. For example, there is a broad distinction between approaches that conceptualize WM as consisting of multiple domain-specific components (e.g., Baddeley, 2007) and those that conceptualize WM as a domain-general system that controls the focus of attention (e.g., Cowan, 2005). Although there are definite structural and conceptual differences between these and related theoretical approaches (see Shah \& Miyake, 1999), it has been recently argued that these differences may be a matter of emphasis rather than being substantive (Logie, 2011). Importantly, whereas theories differ in how they cleave or do not cleave different functions of the WM system into component parts, all agree that higher order cognitive processes are somehow supported by a capacity-limited temporary workspace. The question remains-how might WM support the acquisition and processing of verbal information?

\section{THEORETICAL APPROACHES TO VWM}

Theoretical approaches to VWM attempt to account for real and observable individual differences in language proficiency. A major distinction can be drawn between theories that explain these individual differences with reference to capacity limits inherent in VWM and those that instead argue that apparent capacity limits are emergent properties of differences in long-term linguistic knowledge and architectural limitations on processing linguistic stimuli. Each approach is reviewed in turn.

\section{Capacity-limit theories of VWM}

Two capacity-limit models have dominated theoretical discussion on the role of VWM in language processing-Just and Carpenter
(1992) and Waters and Caplan (1996).* Although fairly old in scientific terms, both have been influential in shaping how current language researchers view the role of VWM in language, including language acquisition researchers investigating both typical and atypical acquisition.

Both Just and Carpenter (1992) and Waters and Caplan (1996) argue that VWM directly constrains language processing, explaining that individual differences in both typical and atypical populations (e.g., adults with aphasia) are caused by variations in VWM capacity. Crucially, both approaches view VWM as conceptually and functionally distinct from long-term memory processes that support linguistic processing (e.g., vocabulary and syntax). In both models, VWM serves as a temporary workspace in which linguistic input is stored and processed. The models differ in the exact architecture of VWM and their broader commitments to different theoretical models of language.

Just and Carpenter (1992) postulate a WM store that mediates storage and processing of verbal stimuli. The capacity limit varies in the general population, which directly constrains comprehension depending on resource availability. The effect of capacity limits is particularly evident when the capacity of the system is stressed, as is the case in syntactically complex sentences. Consider sentences (1) and (2).

(1) The reporter that _ attacked the senator admitted the error.

(2) The reporter that the senator attacked _ admitted the error.

Sentence (1) contains a center-embedded subject relative clause $(\mathrm{RC} ;$... that attacked

*Lewis et al. (2006) have proposed a more recent model of WM in sentence comprehension, but their focus is on explaining complexity effects using general constraints on attention and principles of memory (e.g., rapid retrieval, decay). Because their model does not yet offer an explanation for individual differences (or development), I concentrate on Just and Carpenter (1992) and Waters and Caplan (1996). 
the senator...), whereas sentence (2) contains an object $\mathrm{RC}(\ldots$ that the senator attacked...). A common finding in the adult and child psycholinguistic literature is that, with some qualification, object RCs are more difficult to process than subject RCs (Gibson, 1998; Kidd, 2011). Various explanations for this effect exist, including (i) differences in the distance the displaced element (the reporter) must be moved to its position in the RC (i.e., the gap site) (Frazier, 1987), (ii) greater ambiguity of thematic role assignment in object RCs (Gennari \& MacDonald, 2008; Gibson, 1998), and (iii) the fact that the object-subject-verb (OSV) word order violates the canonical subject-verb-object (SVO) order of English (MacDonald \& Christiansen, 2002). Just and Carpenter (1992) discussed a number of studies in which college-aged adults with high VWM spans processed syntactically complex sentences such as object RCs more efficiently than individuals with low VWM spans. They interpreted this as evidence for a capacity-limit account.

Just and Carpenter (1992) argued further that comprehension is a nonmodular process that is mediated by VWM capacity. That is, all potential sources of information (e.g., syntax, semantics, pragmatics) are incorporated into an analysis of a sentence, provided that VWM capacity is large enough to coordinate all cues to comprehension. In contrast, Caplan and Waters (1999) argued for the division of VWM into two component processes: one devoted to "interpretative" processes such as syntactic analyses of the input and the other devoted to "postinterpretative" processes, such as reanalyzing sentences and making inferences. They argued that most measures of VWM tap deliberate, postinterpretative processes but not more automated interpretative processes typically associated with most forms of sentence comprehension. The approach is modular in the sense that it attempts to isolate syntactic (or syntax-related) processes from other nonsyntactic processes, although exactly how the approach apportions different aspects of comprehension to "interpretative" and "postinterpretative" processes is not entirely clear.
Despite this ambiguity, Caplan and Waters argue that both types of VWM are capacitylimited and therefore directly (and causally) constrain comprehension processes.

\section{Experience-based explanations of VWM}

In contrast to capacity-limit theories, the experience-based theory posits that individual differences in comprehension (and, more broadly, language attainment) are due to the dual influence of (i) differences in experience with different components of language and (ii) architectural limitations on learning and processing. According to this account, VWM is not conceptually distinct from language processing; it is an emergent property of the system itself.

The experience-based account explains the empirically attested relationship between VWM and language comprehension by arguing that measures of VWM are measures of language processing; performance on VWM and language comprehension tasks correlate because they tap the same underlying cognitive process. Consider the most commonly used measure of VWM in studies of language comprehension-Daneman and Carpenter's (1980) VWM span task. In this task, participants read or listen to sentences that vary in length (9-16 words) and are required to (i) judge the sentence as true or false and (ii) remember the final word of each sentence in a set (sets vary from 2 to 6 sentences). At the end of each set, the participant is required to recall each sentence-final word presented in that set. The task requires storage and manipulation of verbal material; however, success clearly depends on many of the same skills required for sentence comprehension. The question that proponents of the experiencebased approach pose is whether the span task taps an independent capacity-limited processing resource (the alternate hypothesis), or whether it is an alternative measure of language processing (their hypothesis).

The experience-based approach is connectionist in nature (Elman et al., 1996); that is, such theorists posit that linguistic knowledge is represented in a distributed fashion across 
a network of interconnected nodes (see Christiansen \& MacDonald, 2009). Different patterns of activation represent different knowledge states (e.g., a subject RC activates a different neural pattern than an object RC), and repeated processing of stimuli leads to increased activation levels in the network pathways that represent knowledge. Knowledge is therefore represented in a graded mannerregularly processed stimuli are more strongly represented than less frequently processed stimuli and are therefore easier to process.

Consider how the experience-based approach explains the asymmetry in processing subject and object RCs. Subject RCs are 2.5 times more common in spoken English than are full (i.e., unreduced) object RCs (Roland, Dick, \& Elman, 2007). As such, a learning mechanism that is sensitive to the frequency with which individual constructional patterns occur in the input predicts the asymmetry. However, the token frequency of each individual constructional pattern might not fully explain the difference. As MacDonald and Christiansen (2002) argued, whereas subject RCs follow English canonical SVO word order (e.g., The suspect that bired the lawyer), object RCs contain the marked and therefore infrequent OSV word order (e.g., The lawyer that the suspect bired). Many English structures follow the SVO pattern, whereas only object RCs and the closely related yet extremely infrequent object cleft constructions (e.g., It was the cat that the dog chased) contain OSV word order. Therefore, the relative frequency of word order patterns is likely to favor subject but not object RC processing. This is because the many structures that follow SVO word order facilitate processing of subject relatives-a regularity effectbecause it creates the expectation that, for instance, a sentence-initial noun is the subject of a sentence. In contrast, as object RCs have no frequent near-neighbors that have the same OSV word order, their processing is contingent on an individual's experience with object RCs alone. MacDonald and Christiansen (2002) refer to this as the frequency $\times$ regularity effect, which captures the idea that experience with an individual structure interacts with word order regularity to predict comprehension difficulty. In the case of subject RCs, the fact that the structure is more common than object RCs and the fact that it has the same SVO word order as highfrequency neighbors such as the simple transitive makes the structure easier to process than object RCs.

Therefore the experience-based approach argues that the distributional properties of the language and an individual's engagement in linguistic endeavors (e.g., reading) lead to differences in the long-term knowledge necessary for the implementation of parsing routines (see also Bybee, 2010). A crucial question concerns the nature of the neurocognitive mechanism that supports the extraction of these regularities. That is, what are the architectural constraints on language learning that predict individual differences, if not VWM? MacDonald and Christiansen (2002) argued that innate differences in phonological representations affect processing, particularly in complex structures such as object RCs. Unexpected word order configurations might place a greater burden on phonological representations, because the serial order of words might need to be kept activated while less frequent parsing routines are implemented (Acheson \& MacDonald, 2009, 2011).* A more recent suggestion has been statistical learning, which has been directly linked to the acquisition and processing of language and which is subject to individual differences (e.g., Conway, Bauernschmidt, Huang, \& Pisoni, 2010; Kidd, 2012; Misyak \& Christiansen, 2012).

Overall, the experience-based approach differs from capacity-limit approaches in that it explains variations in language performance as derived from differences in the long-term representations that support language use,

*This reasoning sounds very similar to capacity-limit arguments; however, it differs in the sense that innate variability in phonological representations constitutes long-term knowledge. See discussion of Acheson, Hamidi, Binder, and Postle (2011) below. 
which are acquired over the course of a speaker's lifetime. The approach is somewhat aligned with approaches to VWM that identify a significant role for LTM in verbal processes (e.g., Cowan, Rouder, Blume, \& Scott Saults, 2012), and models of WM that identify a major role for learning and expertise (and therefore LTM) in performance (Ericsson \& Kintsch, 1995).

\section{PREDICTIONS AND IMPLICATIONS OF EACH APPROACH}

The capacity-limit and experience-based approaches make divergent predictions regarding the role of VWM in language comprehension. Whereas the capacity-limit approaches predict a causal role for VWM, the experiencebased approach predicts that variations in comprehension ability are due to variations in long-term linguistic representations and parsing routines shaped by the language learning process. According to the experiencebased account, VWM effects are largely language processing (and acquisition) effects in disguise.

The two theoretical explanations also differ in how they extend to language acquisition. The conceptual distinction between memory and language made in the capacitylimit approach leads to the logical possibility that growth in one domain is distinct from growth in the other. However, most capacity-limit arguments in the developmental literature argue for a kind of "filter" process, in which VWM is necessary in order for children to analyze their input and therefore acquire linguistic knowledge (e.g., Baddeley, Gathercole, \& Papagno, 1998), parse sentences to deduce innate parametric settings (e.g., Valian, 1991), or integrate multiple sources of information into the parse of a sentence (Felser, Marinis, \& Clahsen, 2003). As such, increases in VWM are argued to lead to more rapid gains in acquisition or in better performance.

The experience-based approach predicts that growth in language knowledge will lead to corresponding gains in performance on measures of VWM by virtue of the fact that VWM tasks measure linguistic language processing skills. The capacity-limit and experience-based approaches therefore make a divergent prediction: all things being equal (e.g., executive function, attention), the capacity-limit approach predicts that increases in VWM will lead to improvements in linguistic knowledge, whereas the experience-based approach predicts that increases in exposure to language predicts improvements in linguistic knowledge and improvements in VWM capacity.

\section{LITERATURE REVIEW}

Previous reviews of the literature investigating the role of VWM in sentence comprehension have been constrained by the fact that very little research had addressed the topic (Cowan, 1996; Montgomery, 1996, 2003), whereas more recent reviews have focused on WM and language at a more general level (Montgomery, Magimaiaj, \& Finney, 2010). A critical mass of studies now exists, which are considered in the current review. Differences in the way in which both VWM and sentence comprehension have been measured in these studies preclude the use of meta-analytic techniques, which would have permitted a quantitative evaluation of the data. Therefore, a narrative review was undertaken instead. As will become evident, most developmental research on VWM and sentence comprehension has followed capacity-limit models in assuming a conceptual distinction between VWM and linguistic knowledge rather than directly testing the predictions of each model.

Studies that have investigated the role of VWM in language comprehension have used both off-line and online reaction time methodologies to assess language comprehension. The off-line studies are reviewed first, followed by the online studies.

\section{Verbal working memory and off-line language comprehension}

Studies that investigate the role of VWM in off-line language comprehension come in two varieties. The first are those studies that test for associations between VWM and 
general (mainly standardized) measures of comprehension. The second variety tests for associations between WVM and particular sentence types, as is typically done in the adult literature. Both types of study are reviewed in turn.

\section{Verbal working memory and general sentence comprehension}

In an early attempt at investigating the role of VWM in comprehension, Daneman and Blennerhassett (1984) developed a listening span task for preliterate children. In this task, 3- to 5-year-old children listened to sets of sentences that contained between 1 and 5 sentences. Participants were required to recall every sentence in a set verbatim. The children's VWM span was determined by how many sentences they could recall in a set. Performance on the modified listening span task predicted sentence comprehension over and above the influence of verbal STM, as measured by digit recall. Although interesting, it is unclear whether the modified task is a genuine measure of complex VWM span as defined by the capacity-limit approach. Sentence repetition taps language processing and production skills in both children and adults (Kidd, Lieven, \& Tomasello, 2006; Potter \& Lombardi, 1990); it is, therefore, as much a measure of long-term linguistic knowledge as immediate memory. Alloway, Gathercole, Willis, and Adams (2004) showed that sentence repetition measured a construct that was related, yet statistically independent of VWM, which they interpreted to be Baddeley's (2000) Episodic Buffer. As we will see, children's sentence repetition may be a key skill that can help decide between the capacity-limit and experience-based models of VWM.

Engel de Abreu, Gathercole, and Martin (2011) investigated the role of VWM in sentence comprehension in 119 typically developing 6-year-old children learning Luxembourgish as a first language. Two measures of VWM from the Automated Working Memory Assessment (Alloway, 2007) were usedcounting recall and backward digit span. Although neither measure explicitly involves language beyond the storage and manipulation of number words, Gathercole, Pickering, Ambridge, and Wearing (2004) showed that both measures loaded onto the same factor as the more typically used listening recall task, suggesting that they tap the same underlying construct. The children's sentence comprehension was tested using the Test for the Reception of Grammar (TROG, Bishop, 2003), a generic test of the comprehension of different grammatical contrasts (e.g., spatial prepositions, negation, passives, RCs). The children's verbal STM also was tested using a nonword repetition task and a digit recall test. The results showed that verbal STM predicted vocabulary knowledge. The relationship between VWM and language was more complicated. An initial analysis showed that verbal STM and non-verbal IQ predicted performance on sentence comprehension; however, the link between verbal STM and comprehension was mediated by vocabulary size. When the authors ran a new set of analyses in which nonverbal IQ was excluded, they found that VWM predicted sentence comprehension over and above the influence of vocabulary. The authors interpreted this result to suggest that the component of VWM that shares variance with fluid intelligence-called controlled processing (Conway, Cowan, Bunting, Therriault, \& Minkoff, 2002)—supports the acquisition and processing of higher order language (i.e., grammar). This result endorses the capacitylimit approach to VWM in sentence comprehension.

In a follow-up study conducted some 3 years later, Engel de Abreu and Gathercole (2012) investigated the role of verbal STM and VWM in the acquisition of vocabulary and grammar in the same children's L1 (Luxembourgish), L2 (German), and L3 (French). The same relations replicated for the children's L1, and identical results were observed for the children's L2. That is, VWM predicted sentence comprehension, as measured by the TROG, over and above the influence of measures of verbal STM, phonological awareness, and (L1) vocabulary. Verbal working memory did not predict sentence comprehension in 
the children's L3, a result that is likely to be due to the fact that the children had only recently begun formal instruction in French within the previous 6 months. The L1 and L2 data suggest that the relationship between VWM and language remains stable in early childhood.

Magimairaj and Montgomery (2012) also investigated the role of VWM in predicting variance in performance on the TROG for sixty-five 6- to 12-year-old English-speaking children. Their study differed in that they manipulated sentence complexity in the VWM task. They used the standard listening span task but with two conditions: (i) in the "simple" VWM task, children heard canonical intransitive or transitive sentences, and (ii) in the "complex" VWM task, the children heard noncanonical object RCs (e.g., the cat that the dog chased). Both VWM measures correlated with performance on the TROG; however, only performance on the simple VWM task significantly predicted TROG scores when both the VWM measures were entered into a linear regression. The authors concluded that simple tasks are more robust measures of children's sentence comprehension because they sufficiently capture controlled attentional focus, a conclusion similar to that of Engel de Abreu et al. (2011). Note, however, that an overlooked explanation that implicates experience concerns linguistic overlap between the VWM tasks and the TROG. The TROG tests a range of grammatical contrasts, but an overwhelming number of test sentences have canonical SVO word order than noncanonical word order.* As such, Magimairaj and Montgomery's results might reflect the topographical overlap

*This is further complicated by the fact that the test is discontinued if children fail five blocks of sentences in a row (total number of blocks $=21$ ). Because the noncanonical sentences occur in blocks 13 (passives) and 21 (object RC), many of the children may not have been tested on them. In contrast, the first 6 blocks ( 24 sentences) are unambiguously SV (O), and the children will almost certainly have been tested on all of these sentences. (i.e., similarity, see Friedman \& Miyake, 2004) between the simple VWM task and the TROG.

Other research on the role of VWM as a factor in general sentence comprehension has involved atypical populations. One group that is particularly relevant for discussions about sentence comprehension is children with specific language impairment (SLI), who have been shown to have deficits in VWM (Ellis Weismer, Evans, \& Hesketh, 1999; Leonard et al., 2007). The exact nature of the deficit depends on the linguistic system being learned. For instance, children acquiring morphologically complex languages such as Hungarian typically experience difficulty storing and manipulating morphologically complex sequences, whereas English-speaking children experience difficulty with syntactically complex sequences (e.g., Marton \& Schwartz, 2003; Marton, Schwartz, Farkas, \& Katsnelson, 2006).

Archibald and Gathercole (2006) reported that children with SLI are nine times more likely to have a VWM deficit than typically developing children. They also reported that the diagnosis of a VWM deficit persisted when the VWM scores of children with SLI were compared against their verbal age, suggesting that the VWM deficit exceeds their language deficit. This result was confirmed by Lum, Conti-Ramsden, Page, and Ullman (2012), who reported a VWM deficit in fifty-one 8- to 11-year-old children with SLI compared with 51 age-matched typically developing children, even after variance attributable to linguistic knowledge was removed (although the difference was significantly attenuated). However, Lum et al. did not find a significant correlation between VWM and grammatical knowledge in either the SLI or typically developing samples. This result is inconsistent with the study of Engel de Abreu et al. (2011), Engel de Abreu and Gathercole (2012), and Magimairaj and Montgomery (2012).

Montgomery $(2000 a, 2000 b)$ reported the first studies to explore the role of VWM in sentence comprehension in children with SLI and matched controls. In both studies, children completed a VWM task and a 
sentence comprehension task. The sentence comprehension task tested sentences containing "nonredundant" and "redundant" information (e.g., "Point to the picture of the cats"nonredundant; "Point to the picture of the three cats" - redundant). Although the group with SLI performed significantly worse than the typically developing age-matched controls on both tasks, the relationship between performance on the VWM and language measures was inconsistent. For instance, in Montgomery (2000a) there was a significant positive correlation between VWM and sentence comprehension in the control children, yet the same correlation was negative for the SLI children, suggesting (paradoxically) that lower VWM led to improved comprehension. In Montgomery (2000b), there were no significant associations between VWM and comprehension.* These inconsistent results might be due to low participant numbers in each group $(\mathrm{n}=12)$, which was likely to render any correlational analysis underpowered.

The general consensus in the literature on SLI is that the VWM deficits found in the disorder are attributable to problems children experience both in the storage and the processing (speed and manipulation of units) of verbal stimuli (Archibald \& Gathercole, 2007; Leonard et al., 2007). This argument accords with a capacity-limit explanation of VWM in development. However, recent findings suggest a role for long-term linguistic knowledge. For instance, Mainela-Arnold, Evans, and Coady (2010) reported that performance on a VWM span task in 8- to 12-year-old children with SLI and typically developing controls was predicted by their semantic knowledge and efficiency in lexical processing (see also Mainela-Arnold \& Evans, 2005). Studies with other special populations also sug-

\footnotetext{
*The correlation between VWM and comprehension was positive and significant in the control group matched with the SLI for receptive vocabulary $(r=.54)$; however, this was not deemed significant following Bonferroni adjustment to control for inflation of Type I error rate following multiple correlational analyses.
}

gest that VWM deficits might be an outcome rather than a cause of language impairment. For instance, Nation, Adams, Bowyer-Crane, and Snowling (1999) argued that poor VWM performance in children with poor reading comprehension reflected deficits in underlying speech and language skills rather than a capacity deficit. Consistent with this argument, Alloway and Gathercole (2005) reported that sentence repetition but not VWM significantly predicted spoken language comprehension and production in a sample of 7- to 11-year-old children with learning difficulties. Because sentence repetition taps into long-term linguistic knowledge accessed during language processing, the result is consistent with predictions of the experience-based account. It is interesting to note that children with poor comprehension skills also experience problems with sentence repetition (Marshall \& Nation, 2003), and that sentence repetition is used to diagnose SLI (Conti-Ramsden, Botting, \& Faragher, 2001).

\section{Verbal working memory and complex sentence comprehension}

Most empirical and theoretical discussions of the role of VWM in language comprehension make a distinction between simple and complex sentences; the assumption of capacity-limit approaches is that complex sentences sufficiently tax processing resources (or the language processor) to implicate VWM. As argued previously, complexity is often confounded with structural frequency, which implicates a person's experience with a structure. Developmental studies that have actively manipulated the structural properties of sentences in comprehension tasks suggest that, consistent with the adult literature, VWM supports only the processing of complex syntactic forms rather than sentence comprehension in general. However, the developmental studies have been hampered by inconsistent definitions of complexity, as well as inconsistent use of different VWM tasks across studies.

Montgomery, Magimairaj, and O'Malley (2008) investigated the role of VWM and processing speed in simple and complex 
sentence comprehension in 52 typically developing 6- to 12-year-old (mean age $=8$ years 8 months) English-speaking children. The children's VWM memory was tested using an "attentional resource control/allocation" task, which is a listening span task that contains both simple and complex sentences. The children's sentence comprehension was tested using a picture selection task, which tested children's comprehension of simple and complex sentences. The simple sentences were active transitives (e.g., The little boy kissed the happy little girl). Two types of "complex" sentence were tested: (i) passives (e.g., The woman was kissed by the baby) and (ii) sentences involving binding principles (e.g., Bugs Bunny says Daffy Duck is bugging bim/bimself). The results showed that VWM significantly predicted complex sentence comprehension over and above the influence of age. Processing speed, as measured by a simple audiovisual matching task (e.g., Touch the green square when you bear "green"), also contributed uniquely to complex sentence performance. The comprehension of simple sentences was not associated with any cognitive measure. Using a slightly different VWM measure devised by Gaulin and Campbell (1994), Montgomery and Evans (2009) tested children with SLI (mean age = 9 years 1 month) and typically developing age-matched (mean age $=9$ years 1 month) and language-matched (mean age $=6$ years 3 months) controls on the same sentence comprehension task. Verbal working memory significantly predicted complex sentence comprehension in both the SLI and languagematched groups but not in the age-matched group.

The results from these two studies are broadly consistent with the proposal that VWM is strongly associated with complex sentence comprehension. However, Montgomery and colleagues' definition of complexity makes it difficult to isolate the source of the association and distinguish between the capacity-limited and experiencebased approaches. They argued that their two complex sentence types were equivalent be- cause they involved "movement" of displaced noun phrases (Chomsky, 1995); however, it is unclear whether the complexity of both sentence types can be reduced to a common underlying process. The difficulty associated with the passive is almost certainly syntactic, but it is unclear whether children's difficulty with the structure is due to the fact that it has noncanonical word order and is therefore low frequency*, as opposed to involving complex derivations that require movement. The difficulty associated with sentences with pronominal reference is at least partially discoursepragmatic (Matthews, Lieven, Theakston, \& Tomasello, 2009; O'Grady, 2005). Therefore, it is unclear whether the two sentence types should be treated as equivalent in terms of their complexity.

Montgomery, Evans, and Gillam (2009) investigated the relationship between VWM and sentence comprehension using Montgomery's (2000a) attentional resource capacity/allocation VWM task and a picture selection task. Once again "simple" and "complex" sentences were tested, and VWM was significantly associated with complex sentence comprehension in children with SLI but not aged-matched controls. However, the results are again ambiguous because of the manner in which sentence complexity was defined. The children were tested on two types of complex sentences: reduced subject RCs that contained either two (e.g., The girl smiling is pushing the little boy) or three clauses (e.g., The boy standing is kissing the little girl sitting). Performance was collapsed across these two sentence types to form one category of complexity. As such, it is unclear whether the children with SLI experienced difficulty with the two-clause sentences, the three-clause sentences, or both. Furthermore, the sentences themselves are highly unusual and probably very infrequent.

*The passive voice is used in less than $1 \%$ of utterances in child-directed speech (Bencini \& Valian, 2008). 
More tightly controlled linguistic stimuli are required to narrow down the relationship between VWM and complex sentence comprehension. Following adult research, studies need to test minimal pairs of sentences that are controlled for length and preferably lexical content. Weighall and Altmann (2011) conducted a study that investigated RC processing in 32 typically developing 6- to 8-yearold children. The children completed a variant of the listening span task (Stothard \& Hulme, 1992) and a picture selection task that tested their comprehension of center-embedded (3) and right-branching (4) subject RCs.

(3) The cat that bumped the bear will hug the cow.

(4) The cow will hug the cat that bumped the bear.

The sentences were presented in two conditions, one in which there was full contextual support for an RC analysis (following Corrêa, 1995) and one in which there was partial contextual support. Following the presentation of each test sentence, the children were asked comprehension questions that probed their understanding of the agent-patient relations in both the main clause and the RC (e.g., Which animal will bug the cow?). Following an analysis strategy used in the adult literature, the sample was divided into "High" and "Low" VWM groups. The High VWM group was more successful overall in the task; however, VWM span interacted with contextual support, sentence type, and question type. For the highspan children, full contextual support facilitated their comprehension of both main and relative clauses; for the low-span children, context facilitated their comprehension of RCs only. These results suggest that high VWM span allowed children to incorporate contextual cues into their processing across a wider array of contexts. Overall, the children experienced most difficulty in processing the main clause in center-embedded sentences. That is, for sentence (3), they had difficulty answering the question Who will bug the cow?, performing at or close to chance. This is consistent with research on younger children (Kidd \& Bavin, 2002), who find sentence processing difficult when a main clause is interrupted by an RC. Overall, the results show that high VWM span is associated with better comprehension in syntactically complex material.

Boyle, Lindell, and Kidd (2013) investigated the role of VWM in 4- to 6-year-old Englishspeaking children's comprehension of four different sentence types. Recognizing the problems in the way complexity had been operationalized in previous studies, they tested two potential sources of complexity: (i) the traditional linguistic notion of subordination (Lust, Foley, \& Dye, 2009) and (ii) canonical versus noncanonical word order. These two potential sources of complexity were fully crossed to produce 4 sentence types that were matched for length and lexical content, as shown in (5)-(8).

(5) The little boy is hugging the girl.

(6) The girl is being hugged by the boy.

(7) The nice boy that is hugging the girl.

(8) The girl that the nice boy is hugging.

Sentences (5) and (6), a simple active and a simple passive, respectively, are monoclausal sentences. In contrast, sentence (7) is a subject RC and sentence (8) is an object RC; both sentences are complex because they involve subordination. The sentences differ on the orthogonal dimension of canonicity: (5) and (7) follow canonical English SVO word order, whereas (6) and (8) have noncanonical word order. Boyle et al. (2013) tested 50 typically developing children's comprehension of these sentence types using a picture pointing task. In addition, the children were tested on three measures of verbal memory: (i) phonological short-term memory, (ii) sentence repetition, and (iii) VWM (backward digit span). On the sentence comprehension task, the children performed almost at ceiling on the simple active and subject RC sentences, suggesting they had no trouble comprehending sentences containing canonical word order. In contrast, the children performed worse on the passives (79\% correct) and the object RCs (59\%), suggesting that noncanonical sentences are more complex for young children. 
The results of a regression analysis revealed that sentence repetition and not VWM predicted children's comprehension of noncanonical sentences. This result is interesting in the context of the capacity-limit and experience-based models of VWM. Sentence repetition is a sensitive measure of children's and adult's language processing (e.g., Potter \& Lombardi, 1990). The method has been used in acquisition research to reveal children's sensitivities to structures that differ in frequency (e.g., Diessel \& Tomasello, 2005; Kidd, Brandt, Lieven, \& Tomasello, 2007; Kidd et al., 2006). Although repetition is naturally capacity limited (implicating some notion of a short-term mental workspace), the method taps into parsing routines and therefore long-term linguistic knowledge. Therefore, the finding of Boyle et al. (2013) that sentence repetition predicts sentence comprehension instead of VWM (measured as backward digit span) provides some support for the experience-based approach. Recall that his is not the only study to report this finding; Alloway and Gathercole (2005) reported the same result in a sample of children with learning difficulties.

In an ancillary analysis, Boyle et al. (2013) showed that removing the sentence repetition variable from the regression equation yielded results consistent with past research, that is, VWM significantly predicted noncanonical sentence repetition. This suggests that the shared variance between sentence repetition and VWM significantly predicts sentence comprehension. Recent neurolinguistic evidence suggests that this might reflect the activation of long-term knowledge involved in language production. Acheson, Hamidi, Binder, and Postle (2011) reported a repetitive transcranial magnetic stimulation study in which they found that stimulation of the posterior superior temporal gyrus interfered with both nonword reading and serial recall of nonwords. They interpreted this result to suggest that the short-term retention of verbal material is accomplished via the phonological encoding component of the speech production system. The posterior superior tem- poral gyrus also appears to be involved in sentence comprehension. In a large MRI study of 210 stroke patients, Leff et al. (2009) found that the integrity of the posterior superior temporal gyrus predicted both serial recall and spoken sentence comprehension. These latter data argue against a capacity-limit approach to verbal STM, in which verbal STM and long-term linguistic knowledge are functionally separate. They instead suggest that verbal STM tasks such as digit recall tap into long-term representations used for language processing.* To my knowledge, comparable studies investigating VWM and sentence comprehension do not yet exist, but this would be an obvious direction for future research.

\section{Verbal working memory and online studies of sentence comprehension}

There is a small literature that has investigated the role of VWM in children's online sentence processing. Montgomery (2000a) found no relationship between VWM and online word monitoring in 9-year-old children with SLI and typically developing controls, but the lack of association might be due to the aforementioned power problems or because word monitoring is not a sensitive enough measure of syntactic processing. Other studies have used more sensitive measures and have found associations between VWM and online processing, but the results are not entirely consistent across studies.

Booth, MacWhinney, and Haraski (2000) investigated the role of VWM in 8- to 11-year-old children's online processing of

\footnotetext{
*An anonymous reviewer asked whether it is possible that these neurolinguistic findings could be interpreted to suggest that the pSTG is part of two brain networks, one supporting phonological encoding (i.e., long-term knowledge) and another supporting a capacity-limited WM space. This interpretation is unlikely. Acheson et al. (2011) used fMRI to identify the target region, in which their participants completed language production tasks only. Therefore, they isolated the pSTG as a brain region supporting long-term knowledge of language but found that rTMS to this region also compromised serial recall of words.
} 
center-embedded subject and object RCs (see sentences 1 and 2 previously). Two different but complementary methods were used: (i) self-paced reading and (ii) self-paced listening. Verbal working memory was tested using a variant of the reading span task, and short-term memory for verbal material was measured using (forward) digit span. Across both experiments, Booth et al. found that verbal STM and not VWM was associated with online processing at points of high sentence complexity (boundary between relative and main clauses). Verbal working memory predicted off-line comprehension of the test questions in the reading but not the listening task, which was instead predicted by verbal STM. This is suggestive of a modality effect; however, the result is inconsistent with the findings that VWM and not short-term verbal storage is associated with off-line spoken sentence comprehension (e.g., Engel de Abreu et al., 2011; Engel de Abreu \& Gathercole, 2012; Montgomery, 1995; Montgomery et al., 2008; Weighall \& Altmann, 2011). Interestingly, Arosio, Guasti, and Stucchi (2011) reported that neither verbal STM nor VWM was associated with online processing of subject and object RCs in Italian-speaking 9-year-olds in a selfpaced listening task, and that verbal STM as measured by forward digit span predicted offline comprehension of particularly complex object RCs. Future research that builds on these studies is clearly needed.

Two other studies using online methodologies have found effects of VWM in children's on-line processing. Felser et al. (2003) reported that differences in VWM span were associated with the use of different strategies when resolving sentences that contained RC attachment ambiguities (e.g., The busband of the actress who was on the balcony). Finally, Roberts, Marinis, Felser, and Clahsen (2007) reported that adults and 5- to 7-year-old children with high VWM spans processed complex filler-gap dependencies more efficiently than same age peers with lower VWM spans. However, the effect was only marginally significant, and the authors did not report individual analyses of the child and adult high
VWM span groups, making it difficult to ascertain the strength of the effect in each group. This may represent a problem with statistical power, because reaction times are notoriously messy and the group sizes were small. Future studies should aim to test greater numbers of children.

\section{CONCLUSIONS AND RECOMMENDATIONS}

Verbal working memory and sentence comprehension is an intriguing area. Language acquisition and language processing must be supported by a neurocognitive mechanism that enables the retention and analysis of language. Working memory is arguably the most intensively studied construct in cognitive psychology, and it has generally been assumed that it plays some role in psycholinguistic processes. However, this assumption has largely resulted in research that takes a capacity-limit WM system as a theoretical primitive, without seriously considering the experience-based argument that VWM is an emergent property of long-term linguistic knowledge.

What kind of data are needed? First, we need experimental designs that explicitly test competing theoretical accounts (see examples from the adult literature: Misyak \& Christiansen, 2012; Wells, Christiansen, Race, Acheson, \& MacDonald, 2009). In developmental research, the gold standard is studies that utilize a longitudinal design, which are notably absent in studies of VWM and sentence comprehension. In a closely related area, a recent longitudinal study by MelbyLervåg et al. (2012) reported data that contradicts the long-held belief that verbal STM, as measured by nonword repetition, causally predicts vocabulary acquisition (cf. Baddeley et al., 1998). The authors reported no longitudinal relationship between nonword repetition and subsequent vocabulary acquisition. A potential shortcoming of this study is that it followed children from 4 to 8 years of age; it is possible that STM is predictive of vocabulary acquisition at earlier ages. Likewise, it is possible that VWM is involved in the 
acquisition of complex structures during the initial stages of acquisition and not once children have mastery over a structure. That is, the capacity-limited and experience-based approaches might be relevant in explaining acquisition at different stages of the acquisition process and therefore may not be mutually exclusive.

Training studies that target growth in both VWM and language are also required. Both WM and language have been shown to respond well to training: WM training programs have been shown to be effective (Holmes, Gathercole, \& Dunning, 2009; St. Clair-Thompson, Stevens, Hunt, \& Bolder, 2010), and book reading has been found to significantly improve sentence comprehension (Vasilyeva, Huttenlocher, \& Waterfall, 2006). This means that causal models about the direction of the VWM and sentence comprehension relationship could be teased apart in studies that compared both kinds of training against appropriate controls.

Of course, longitudinal and training studies can be costly, and many questions can be answered using cross-sectional and comparative designs. Progress in the field also has been hampered slightly by the use of many different VWM measures, which often makes results across studies difficult to compare. Future studies should aim to use multiple and psychometrically validated measures (e.g., the Automated Working Memory Assessment; Al- loway, 2007). In addition, care must be taken when selecting linguistic items for testing sentence comprehension. The literature points to the conclusion that VWM could be implicated in some way in the comprehension of complex syntactic forms, but complexity must be carefully operationalized. Future studies need tighter linguistic controls.

The past 10 years have marked the emergence of research that has investigated the relationship between VWM and sentence comprehension. In many senses, the field is still rather fragmented: different researchers concentrate on different populations of children (e.g., typically developing, children with SLI, poor comprehenders), and as such, their research goals have not always been the same. The results thus far have been mixed. It is notable that most of the research has been conducted with special populations. Although these studies almost always have typically developing control groups, their sample sizes are invariably too small to conduct the kind of individual differences analyses that would allow firm conclusions to be drawn regarding the competing theoretical accounts of VWM in sentence comprehension (and language acquisition in general). It is promising to see that larger studies are now starting to come online (e.g., Engle de Abrue, 2011, 2012), moving the field closer to a nuanced understanding of the relationship between VWM and sentence comprehension.

\section{REFERENCES}

Acheson, D. J., Hamidi, M., Binder, J. R., \& Postle, B. R. (2011). A common neural substrate for language production and verbal working memory. Journal of Cognitive Neuroscience, 23, 1358-1367.

Acheson, D., \& MacDonald, M. (2009). Verbal working memory and language production: Common approaches to serial ordering of verbal information. Psychological Bulletin, 135, 50-68.

Acheson, D. J., \& MacDonald, M. C. (2011). The rhymes that the reader perused confused the meaning: Phonological effects during on-line sentence comprehension. Journal of Memory and Language, 65, 193207.

Alloway, T. P. (2007). Automated working memory assessment. London: Pearson.
Alloway, T. P., \& Gathercole, S. (2005). The role of sentence recall in reading and language skills of children with learning difficulties. Learning and Individual Differences, 15, 271-282.

Alloway, T. P., Gathercole, S. E., Willis, C., \& Adams, A.-M. (2004). A structural analysis of working memory and related cognitive skills in young children. Journal of Experimental Child Psychology, 87(2), 85-106.

Archibald, L. M. D., \& Gathercole, S. E. (2006). Shortterm and working memory in specific language impairment. International Journal of Language \& Communication Disorders, 41, 675-693.

Archibald, L. M. D., \& Gathercole, S. E. (2007). The complexities of complex memory span: Storage and processing deficits in specific language 
impairment. Journal of Memory and Language, 57, 177-194.

Arosio, F., Guasti, M. T., \& Stucchi, N. (2011). Disambiguating information and memory resources in children's processing of Italian relative clauses. Journal of Psycholinguistic Research, 40, 137-154.

Baddeley, A. (2000). The episodic buffer: A new component of working memory? Trends in Cognitive Sciences, 4, 417-423.

Baddeley, A. (2007). Working memory, thought, and action. New York, NY: Oxford University Press.

Baddeley, A. D., Gathercole, S. E., \& Papagno, C. (1998). The phonological loop as a language learning device. Psychological Review, 105, 158-173.

Bybee, J. (2010). Language, usage and cognition. Cambridge: Cambridge University Press.

Bishop, D. (2003). Test for the reception of grammar (2nd ed.). Oxford: Harcourt Assessment.

Booth, J. R., MacWhinney, B., \& Haraskai, Y. (2000). Developmental differences in visual and auditory processing of complex sentences. Child Development, 71, 981-1003.

Boyle, W., Lindell, A. K., \& Kidd, E. (2013). Investigating the role of verbal working memory in young children's sentence comprehension. Language Learning, 63, 211-242.

Bencini, G. M. L., \& Valian, V. (2008). Abstract sentence representation in 3-year-olds: Evidence from comprehension and production. Journal of Memory and Language, 59, 97-113.

Caplan, D., \& Waters, G. S. (1999). Verbal working memory and sentence comprehension. The Behavioral and Brain Sciences, 22, 77-126.

Christiansen, M. H., \& MacDonald, M. C. (2009). A usagebased approach to recursion in sentence processing. Language Learning, 59(Suppl. 1), 126-161.

Chomsky, N. (1995). The minimalist program. Cambridge, MA: The MIT Press.

Conti-Ramsden, G. M., Botting, N. F., \& Faragher, B. (2001). Psycholinguistic markers for specific language impairment (SLI). Journal of Child Psychology and Psychiatry Allied Disciplines, 42, 741-748.

Conway, A. R., Cowan, N., Bunting, M. F., Therriault, D. J., \& Minkoff, S. R. B. (2002). A latent variable analysis of working memory capacity, short-term memory capacity, processing speed, and general fluid intelligence. Intelligence, 30, 163-183.

Conway, C. M., Bauernschmidt, A., Huang, S. S., \& Pisoni, D. B. (2010). Implicit statistical learning in language processing: Word predictability is the key. Cognition, 114, 356-371.

Corrêa, L. (1995). An alternative assessment of children's comprehension of relative clauses. Journal of Psycholinguistic Research, 24, 183-203.

Cowan, N. (1996). Short-term memory, working memory, and their importance in language processing. Topics in Language Disorders, 17, 1-18.
Cowan, N. (2005). Working memory capacity. Hove, UK: Psychology Press.

Cowan, N., Rouder, J. N., Blume, C. L., \& Scott Saults, J. (2012). Models of verbal working memory capacity: What does it take to make them work? Psychological Review. doi:10.1037/a0027791

Daneman, M., \& Blennerhassett, A. (1984). How to assess the listening comprehension skills of prereaders. Journal of Educational Psychology, 76, 1372-1381.

Daneman, M., \& Carpenter, P. A. (1980). Individual differences in working memory and reading. Journal of Verbal Learning and Verbal Behavior, 19, 450-466.

Diessel, H., \& Tomasello, M. (2005). A new look at the acquisition of relative clauses. Language, 81, 1-25.

Ellis Weismer, S., Evans, J., \& Hesketh, L. (1999). An examination of verbal working memory capacity in children with specific language impairment. Journal of Speech. Language and Hearing Research, 42, 1249-1260.

Elman, J., Bates, E., Johnson, M., Karmiloff-Smith, A., Parisi, D., \& Plunkett, K. (1996). Rethinking innateness: A connectionist perspective on development. Cambridge, MA: The MIT Press.

Engel de Abreu, P. M. J., \& Gathercole, S. E. (2012). Executive and phonological processes in second language acquisition. Journal of Educational Psychology, 104, 974-986.

Engel de Abreu, P. M. J., Gathercole, S. E., \& Martin, R. (2011). Disentangling the relationship between working memory and language: The roles of short-term storage and cognitive control. Learning and Individual Differences, 21, 569-574.

Ericsson, K. A., \& Kintsch, W. (1995). Long-term working memory. Psychological Review, 102, 211-245.

Felser, C., Marinis, T., \& Clahsen, H. (2003). Children's processing of ambiguous sentences: A study of relative clause attachment. Language Acquisition, 11, 127163.

Frazier, L. (1987). Sentence processing: A tutorial review. In M. Coltheart (Ed.), Attention and performance XII: The psychology of reading (pp. 559-586). Hillsdale, NJ: Erlbaum.

Friedman, N. P., \& Miyake, A. (2004). The relations among inhibition and interference cognitive functions: A latent variable analysis. Journal of Experimental Psychology: General, 133, 101-135.

Gathercole, S., Pickering, S., Ambridge, B., \& Wearing, H. (2004). The structure of working memory from 4 to 15 years of age. Developmental Psychology, 40, 177-190.

Gathercole, S. E., Willis, C., Emslie, H., \& Baddeley, A. (1992). Phonological memory and vocabulary development during the early school years: A longitudinal study. Developmental Psychology, 28, 887-898.

Gibson, E. (1998). Linguistic complexity: Locality of syntactic dependencies. Cognition, 68, 1-76.

Gennari, S. P., \& MacDonald, M. C. (2008). Semantic indeterminacy in object relative clauses. Journal of Memory and Language, 58, 161-187. 
Gaulin, C., \& Campbell, T. (1994). Procedure for assessing verbal working memory in normal school-age children: Some preliminary data. Perceptual and Motor Skills, 79, 55-64.

Holmes, J., Gathercole, S. E., \& Dunning, D. L. (2009). Adaptive training leads to sustained enhancement of poor working memory in children, Developmental Science, 12, F9-F15.

Just, M. A., \& Carpenter, P. A. (1992). A capacity theory of comprehension: Individual differences in working memory. Psychological Review, 99, 122-149.

Kidd, E. (Ed.) (2011). The acquisition of relative clauses: Processing, typology, and function. Amsterdam: John Benjamins.

Kidd, E. (2012). Implicit statistical learning is directly associated with the acquisition of syntax. Developmental Psychology, 48, 171-184.

Kidd, E., \& Bavin, E. L. (2002). English-speaking children's understanding of relative clauses: Evidence for general-cognitive and language-specific constraints on development. Journal of Psycholinguistic Research, 31, 599-617.

Kidd, E., Brandt, S., Lieven, E., \& Tomasello, M. (2007). Object relatives made easy: A cross-linguistic comparison of the constraints influencing young children's processing of relative clauses. Language and Cognitive Processes, 22, 860-897.

Kidd, E., Lieven, E., \& Tomasello, M. (2006). Examining the role of lexical frequency in children's acquisition of sentential complements. Cognitive Development, 21, 93-107.

Leff, A. P., Schofield, T. M., Crinion, J. T., Seghier, M. L., Grogan, A., Green, D. W., et al. (2009). The left superior temporal gyrus is a shared substrate for auditory short-term memory and speech comprehension: evidence from 210 patients with stroke. Brain, 132, 3401-3410.

Leonard, L. B., Ellis Weismer, S., Miller, C. A., Francis, D. J., Tomblin, J. B., \& Kail, R. V. (2007). Speed of processing, working memory, and language impairment in children. Journal of Speech, Language, and Hearing Research, 50, 408-428.

Lewis, R. L., Vasishth, S., \& Van Dyke, J. A. (2006). Computational principles of working memory in sentence comprehension. Trends in Cognitive Sciences, 10, 447-454.

Logie, R. H. (2011). The functional organization and capacity limits of working memory. Current Directions in Psychological Science, 20, 240-245.

Lum, J. A., Conti-Ramsden, G., Page, D., \& Ullman, M. T. (2012). Working, declarative and procedural memory in specific language impairment. Cortex, 48, 11381154 .

Lust, B., Foley, C., \& Dye, C. (2009). The first language acquisition of complex sentences. In E. Bavin (Ed.), The Cambridge handbook of child language (pp. 237-257). New York, NY: Cambridge University Press.
MacDonald, M., \& Christiansen, M. (2002). Reassessing working memory: Comment on Just and Carpenter (1992) and Waters and Caplan (1996). Psychological Review, 109, 35-54.

Magimairaj, B. M., \& Montgomery, J. W. (2012). Children's verbal working memory: Role of processing complexity in predicting spoken sentence comprehension. Journal of Speech, Language, and Hearing Research, 55, 669-682.

Mainela-Arnold, E., \& Evans, J. L. (2005). Beyond capacity limitations: Determinants of word recall performance on verbal working memory span tasks in children with SLI. Journal of Speech, Language, and Hearing Research, 48, 897-909.

Mainela-Arnold, E., Evans, J. L., \& Coady, J. (2010). Beyond capacity limitations II: Effects of lexical processes on word recall in verbal working memory tasks in children with and without specific language impairment. Journal of Speech, Language, and Hearing Research, 53, 1656-1672.

Marshall, C., \& Nation, K. (2003). Individual differences in semantic and structural errors in children's memory for sentences. Educational and Child Psychology, 20, 7-18.

Marton, K., \& Schwartz, R. G. (2003). Working memory capacity and language processes in children with specific language impairment. Journal of Speech, Language, and Hearing Research, 46, 1138-1153.

Marton, K., Schwartz, R. G., Farkas, L., \& Katsnelson, V. (2006). Effect of sentence length and complexity on working memory performance in Hungarian children with specific language impairment (SLI): a cross-linguistic comparison. International Journal of Language and Communication Disorders, 41, 653-673.

Matthews, D., Lieven, E., Theakston, A., \& Tomasello, M. (2009). Pronoun co-referencing errors: Challenges for generativist and usage-based accounts. Cognitive Linguistics, 20, 599-626.

Melby-Lervåg, M., Lervåg, A., Lyster, S.-A. H., Klem, M., Hagtvet, B., \& Hulme, C. (2012). Nonword-repetition ability does not appear to be a causal influence on children's vocabulary development. Psychological Science, 23(10), 1092-1098.

Misyak, J. B., \& Christiansen, M. H. (2012). Statistical learning and language: An individual differences study. Language Learning, 62, 302-331.

Montgomery, J. W., Magimairaj, B. M., \& Finney, M. C. (2010). Working memory and specific language impairment: An update on the relation and perspectives on assessment and treatment. American Journal of Speech-Language Patbology, 19, 78-94.

Montgomery, J. W. (1996). Sentence comprehension and working memory in children with specific language impairment. Topics in Language Disorders, 17, 1932.

Montgomery, J. (2000a). Relation of working memory to off-line and real-time sentence processing in 
children with specific language impairment. Applied Psycholinguistics, 21, 117-148.

Montgomery, J. (2000b). Verbal working memory and sentence comprehension in children with specific language impairment. Journal of Speech, Language, and Hearing Research, 43, 239-308.

Montgomery, J. (2003). Working memory and comprehension in children with specific language impairment: what we know so far. Journal of Communication Disorders, 36, 221-231.

Montgomery, J. W., \& Evans, J. L. (2009). Complex sentence processing and working memory in children with specific language impairment. Journal of Speech, Language, and Hearing Research, 52(2), 269-288.

Montgomery, J. W., Evans, J. L., \& Gillam, R. (2009). Relation of auditory attention on complex sentence comprehension in children with specific language impairment: A preliminary study. Applied Psycholinguistics, 30, 123-151.

Montgomery, J. (1995). Sentence comprehension in children with specific language impairment: The role of phonological working memory. Journal of Speech and Hearing Research, 38, 187-199.

Montgomery, J. W., Magimairaj, B., \& O'Malley, M. (2008). The role of working memory in typically developing children's complex sentence comprehension. Journal of Psycholinguistic Research, 37, 331-354.

Nation, K., Adams, J. W., Bowyer-Crane, C. A., \& Snowling, M. J. (1999). Working memory deficits in poor comprehenders reflect underlying language impairments. Journal of Experimental Child Psychology, 73, 139-158.

Oberauer, K., \& Lewandowsky, S. (2010). Modeling working memory: A computational implementation of the Time-Based Resource Sharing theory. Psychonomic Bulletin \& Review, 18, 10-45.

O'Grady, W. (2005). Syntactic carpentry: An emergentist approach to syntax. Mahwah, NJ: Lawrence Erlbaum.

Potter, M. C., \& Lombardi, L. (1990). Regeneration in short-term recall of sentences. Journal of Memory and Language, 29, 633-654.

Roberts, L., Marinis, T., Felser, C., \& Clahsen, H. (2007). Antecedent priming at trace positions in children's sentence processing. Journal of Psycholinguistic Research, 36, 175-188.

Roland, D., Dick, F., \& Elman, J. (2007). Frequency of basic English grammatical structures: A corpus analysis. Journal of Memory and Language, 57, 348-379.

Shah, P., \& Miyake, A. (1999). Models of working memory. In A. Miyake, Shah P. (Eds.), Models of working memory: Mechanisms of active maintenance and executive control (pp. 1-27). New York, NY: Cambridge University Press.

St. Clair-Thompson, H., Stevens, R., Hunt, A., \& Bolder, E. (2010). Improving children's working memory and classroom performance. Educational Psychology, 30, 203-219.

Stokes, S. F., \& Klee, T. (2009). Factors that influence vocabulary development in two-year-old children. Journal of Child Psychology and Psychiatry and Allied Disciplines, 50, 498-505.

Stothard, S. E. and Hulme, C. (1992). Reading comprehension difficulties in children: The role of language comprehension and working memory skills. Reading and Writing, 4, 245-256.

Valian, V. (1991). Syntactic subjects in the early speech of American and Italian children. Cognition, 40, 21-81.

Vasilyeva, M., Huttenlocher, J., \& Waterfall, H. (2006). Effect of language intervention on syntactic skill levels in preschoolers. Developmental Psychology, 42, 164174.

Waters, G., \& Caplan, D. (1996). The capacity theory of sentence comprehension: Critique of Just and Carpenter (1992). Psychological Review, 103, 761-772.

Weighall, A. R., \& Altmann, G. T. M. (2011). The role of working memory and contextual constraints in children's processing of relative clauses. Journal of Child Language, 38, 579-605.

Wells, J., Christiansen, M., Race, D., Acheson, D., \& MacDonald, M. (2009). Experience and sentence processing: Statistical learning and relative clause comprehension. Cognitive Psychology, 58, 250-271.

Willis, C., \& Gathercole, S. (2001). Phonological shortterm memory contributions to sentence processing in young children. Memory, 9, 349-363. 